\title{
Periosteal cells are a major source of soft callus in bone fracture( Dissertation_全文)
}

$\operatorname{AUTHOR}(S)$ :

Murao, Hiroki

\section{CITATION:}

Murao, Hiroki. Periosteal cells are a major source of soft callus in bone fracture. 京都大学, 2014 , 博士(医学)

ISSUE DATE:

2014-07-23

URL:

https://doi.org/10.14989/doctor.k18510

\section{RIGHT:}

The final publication is available at link.springer.com; 平成25年7月1日 発行 Journal of Bone and Mineral Metabolism 第31巻第4号 390頁 ～398頁掲載 
Periosteal cells are a major source of soft callus in bone fracture

Hiroki Murao* • Koji Yamamoto* • Shuichi Matsuda • Haruhiko Akiyama

H. Murao $・$ K. Yamamoto $\cdot$ S. Matsuda $\cdot$ H. Akiyama

Department of Orthopaedics, Kyoto University, 54 Kawahara-cho, Shogoin, Sakyo, Kyoto, Japan

*These two authors contributed equally to this paper.

Corresponding author.

H. Akiyama: Department of Orthopaedics, Kyoto University, 54 Kawahara-cho, Shogoin, Sakyo, Kyoto, Japan. Tel.: +81 75751 3652; Fax: +81 757518409 .

Email: hakiyama@kuhp.kyoto-u.ac.jp

Keywords: Fracture healing $\cdot$ Periosteal cells $\cdot$ Soft callus $\cdot$ Cell lineage $\cdot$ Sox9 


\section{Abstract}

During the healing process after bone fracture, soft callus forms adjacent to the fracture site, is replaced by hard callus, and is finally remodeled to the original bone configuration. Although the cambium layer of the periosteum is reported to play an essential role in callus formation, we still lack direct in vivo evidence of this. To investigate the cell lineage of the soft callus, we analyzed the process of fracture healing in Prxl-Cre;ROSA26 reporter (R26R), Collal(3.6 kb)-Cre;R26R, Colla1(2.3 kb)Cre;R26R, Sox9-CreERT2;R26R, and Sox9-LacZ mice with X-gal staining. In the Prx1Cre;R26R, in which the cells of the periosteum stained for X-gal before fracture, all cells in the soft callus were X-gal positive, whereas in the Collal(3.6 kb)-Cre;R26R mice, the cells in the periosteum before fracture stained for X-gal and the soft callus was partly composed of X-gal-positive cells. In contrast, in the Collal(2.3 $\mathrm{kb})$ Cre; $R 26 R$ mice, in which the mature osteoblasts in the cambium layer of the periosteum were marked before fracture, no cells in the soft callus at the fracture site were X-gal positive. These results suggest that most of the cells in the soft callus are derived from the mesenchymal progenitors in the periosteum, and not from mature osteoblastic cells. Interestingly, in the Sox9-LacZ mice, Sox9-expressing X-gal-positive cells emerged in the periosteum adjacent to the fracture site three days after fracture. We demonstrated this by injecting tamoxifen into the Sox9-CreERT2;R26R mice for three days after fracture, so that these Sox9-expressing periosteal cells gave rise to cells in the soft and hard calli. Our findings show that the periosteal cells in which Sox9 expression is induced just after fracture are the major source of the chondrocytes and osteoblasts in the fracture callus. 


\section{Introduction}

Bone is a highly organized and specialized connective tissue and is composed of two structural types, cortical and cancellous bone. The external and internal surfaces of the cortical bone are covered by the periosteum and endosteum, respectively. Microscopically, the periosteum is composed of an outer fibrous layer containing cells that synthesize collagen fibers and an inner cambium layer consisting of osteoblasts, fibroblasts, and mesenchymal progenitor cells with the capacity to differentiate into osteoblasts and chondrocytes [1]. The spaces in cancellous bone are filled with various types of marrow cells, including pluripotent marrow stromal cells.

Broken bones undergo processes of endochondral ossification and intramembranous ossification that recapitulate certain aspects of skeletal development. The fracture healing process is partitioned into four stages based on histological observations [2]. The inflammatory stage begins immediately after the bone is fractured, in association with the disruption of the surrounding soft tissues, bleeding within the fracture area, and distortion of the marrow architecture, which lead to local inflammation and the formation of a hematoma. At this stage, stem cells originating from multiple sources are likely to be committed to and initiate the cell lineages involved in bone repair and vascularization. At the stage of soft callus formation, the adjacent periosteum becomes thickened and cartilage tissues appear outside the bone. Discrete cartilaginous regions grow progressively and bridge the fractured bone fragments. This cartilaginous template of the soft callus is then replaced by bone, resulting in the formation of a hard callus (the hard callus formation stage). The hard callus is remodeled by osteoclasts and osteoblasts and returns to the original cortical and 
trabecular bone configuration (the bone remodeling stage).

Several possible sources of the cells that produce bone and cartilage at the fracture site have been identified. Although previous studies have reported potential sources of stem cells or progenitor cells in the bone marrow, endosteum, vessel walls, surrounding soft tissues (including muscle and adipose tissues), and the circulation, the periosteum plays a crucial role in fracture healing [3-5]. Periosteal damage or removal results in delayed healing of the fracture [6]. Colnot showed that the periosteum is a major source of osteoblasts and chondrocytes during fracture healing [7]. In particular, the cambium layer is the component of the periosteum essential for bone formation. Ito et al. cultured rabbit periosteal explants and showed that the chondrocyte precursors were located in the cambium layer of the periosteum and that chondrogenesis took place in the juxtaosseous area in the cambium layer [8]. However, we still lack direct in vivo evidence of the progenitor cells in the periosteum and their contribution to fracture healing.

The transcription factor $\operatorname{Sox} 9$ is expressed in all chondroprogenitor cells and plays an essential role in chondrogenesis [9]. Cell fate mapping in Sox9-Cre;ROSA26 reporter $(R 26 R)$ mice revealed that Sox9-expressing limb bud mesenchymal cells give rise to both chondrocytes and osteoblasts, indicating that Sox9 defines osteochondroprogenitor cells during skeletogenesis [10]. We also generated a mouse strain that inducibly expresses Cre recombinase in Sox9-expressing cells [11]. An IRESCreERT2-pA cassette was inserted into the 3' untranslated region of the $\operatorname{Sox} 9$ gene, resulting in Cre recombinase expression directly under $\operatorname{Sox} 9$ cis-regulatory control via the production of a bicistronic Sox9-Cre recombinase mRNA. The administration of tamoxifen to offspring of crosses between Sox9-CreERT2 and $R 26 R$ mice efficiently 
induced the expression of the Cre reporter in Sox9-expressing cells. The advantage of this genetic system in examining gene expression patterns is that the $R 26 R$ strain provides a permanent lineage record through its constitutive X-gal expression in cells that express Cre recombinase, even transiently, in the precursor cells of different cell lineages. Therefore, this mouse line has been useful for tracing Sox9-expressing osteochondroprogenitor cells in vivo. In this study, using several Cre drivers, we found that osteochondroprogenitor cells are initiated in the periosteum adjacent to the fracture site and are the major source of the soft callus.

\section{Materials and methods}

Animals

Prx1-Cre mice, Collal(2.3 kb)-Cre mice, Sox9-LacZ knock-in mice, Sox9CreERT2 mice, and R26R mice have been reported previously [11-15]. Collal(3.6 kb)Cre mice were purchased from the Jackson Laboratory.

Tibia fracture model

Eight-week-old Prx1-Cre;R26R, Collal(3.6 kb)-Cre;R26R, Collal(2.3 kb)Cre;R26R, Sox9-LacZ, and Sox9-CreERT2;R26R mice were used to prepare the tibia fractures. Each mouse was anesthetized with $50-100 \mathrm{mg} / \mathrm{kg}$ pentobarbital administered intraperitoneally. The skin, subcutaneous tissues, and fascia were incised along the anterior part of the left leg and retracted medially, and the tibia was exposed. The middiaphysis of the tibia was fractured using scissors, without stripping off the periosteum. Three to five animals were analyzed for each mouse line. 


\section{Histological analysis}

The fractured tibiae were collected $3,5,7,14,21$, or 28 days after surgery as indicated in each mouse line. The fresh tibia samples were quickly embedded in OCT compound medium (Sakura Finetek) for cryosectioning and sectioned at a thickness of $8 \mu \mathrm{m}$. After the sectioned samples were fixed in $0.2 \%$ glutaraldehyde $/ 2 \%$ formaldehyde, they were stained by $0.15 \%(\mathrm{w} / \mathrm{v})$ Alcian blue $(\mathrm{pH} 2.5)$ and hematoxylin/eosin or with X-gal solution overnight at $37{ }^{\circ} \mathrm{C}$ and counterstained with Nuclear Fast Red as described previously [16].

\section{Results}

Process of fracture healing in mouse tibia

We first clarified the process of callus formation during the healing of mouse tibia fractures using histology with Alcian blue and hematoxylin/eosin staining (Fig. 1a and $b$ ). On day 3 after surgery, the periosteum adjacent to the fracture site started to thicken. On day 7, soft callus formation was distinguished by Alcian blue staining, and the soft callus was replaced by bone to form the hard callus. On day 14, the soft callus had diminished, and a large part of the callus was occupied by hard callus. On days 21 and 28 , the hard callus was remodeled, and newly formed periosteum and bone marrow were detected.

Cells derived from Prxl-promoter-active pluripotent mesenchymal cells and Collal(3.6 $\mathrm{kb}$ )-promoter-active premature mesenchymal cells predominantly contribute to soft callus formation in fracture healing 
We clarified the cell lineage of the soft callus using Prxl-Cre;R26R mice. Because Prx 1 is expressed in the pluripotent mesenchymal cells of mouse limb buds as early as E9.0, before either an osteoblastic or a chondrogenic lineage has been separated [12], all mesenchymal tissues are derived from Prxl-expressing pluripotent cells. On day 0 before fracture, X-gal-positive cells were found in the periosteum, cortical bone, and endosteum. A small number of X-gal-positive cells were also detected in the bone marrow of the diaphysis (Fig. 2a and b). The cells in the growth plate and metaphyseal bones were X-gal positive. On day 7 after surgery, all cells in the soft callus were X-gal positive (Fig. 2c and d), and on day 14, all the cells in both the soft callus and hard callus were stained for X-gal (Fig. 2e and f). On days 21 and 28, X-gal-positive cells were localized in the remodeled bones and the newly formed periosteum (Fig. 2g-j). Thus, all the cells in the callus may have derived from cells that originated from Prxlexpressing mesenchymal cells and that localized in the periosteum, endosteum, and/or bone marrow.

Next, we induced fractures in the tibiae of Collal $(3.6 \mathrm{~kb})-C r e ; R 26 R$ mice, in which the Collal $(3.6 \mathrm{~kb})$ promoter is active in premature mesenchymal cells and osteoblastic cells. On day 0 before fracture, X-gal-positive cells were found in the periosteum, cortical bone, and endosteum. A small number of X-gal-positive cells were also detected in the bone marrow of the diaphysis (Fig. 3a and b). The cells in the metaphyseal bones were X-gal positive. On day 7 after surgery, a large number of Xgal-positive cells dramatically emerged in the thickened periosteum adjacent to the fracture site, and a proportion of the cells in the soft callus stained for X-gal (Fig. 3c-e). On days 14 and 21, most cells in the soft and hard calli and in the newly formed periosteum were X-gal positive (Fig.3f-i). On day 28 after surgery, X-gal-positive cells 
were localized in the remodeled bones (Fig. 3j). These findings suggest that although Collal $(3.6 \mathrm{~kb})$-expressing cells located in the periosteum, endosteum, and/or bone marrow may contribute to the formation of the soft callus, a proportion of the cells in the soft callus are probably derived from Collal $(3.6 \mathrm{~kb})$-promoter-active mesenchymal progenitors in the periosteum adjacent to the fracture site.

Mature osteoblasts in the periosteum and endosteum do not give rise to chondrocytes in the soft callus

To identify the cells in the periosteum that contribute to the formation of the soft callus, we marked mature osteoblasts using Collal $(2.3 \mathrm{~kb})$-Cre; $R 26 R$ mice. On day 0 before fracture, X-gal-positive cells were detected in the cambium layer of the periosteum and endosteum, indicating that they were mature osteoblasts (Fig. 4a and b). The cells in the cortex and a small number of cells in the metaphyseal bones were X-gal positive. However, in contrast, there were no X-gal-positive cells in the bone marrow of the diaphysis. On days 7 and 14 after surgery, a large number of X-gal-positive cells were detected in the hard callus but not in the soft callus (Fig. 4c-f). On days 21 and 28, X-gal-positive cells were detected in the remodeled bones (Fig. 4g-j). These results indicate that the Collal $(2.3 \mathrm{~kb}$ )-expressing mature osteoblasts in the periosteum and endosteum did not give rise to the cells in the soft callus.

Sox9-expressing cells emerge transiently in the periosteum adjacent to the fracture site Our previous approaches based on mouse genetics have demonstrated that Sox9 defines osteochondroprogenitor cells and that Sox9 is essential for chondrogenesis $[9,10]$. Therefore, Sox 9 expression should be induced in the cells that differentiate into 
chondrocytes in the soft callus. To investigate Sox 9 expression closely during an early stage of fracture healing, we induced fractures in Sox9-LacZ knock-in mice [14]. On day 0 before surgery, there were no X-gal-positive cells in the bones, including in the periosteum, endosteum, and bone marrow (Fig. 5a and b). In contrast, the growth plate chondrocytes were X-gal positive. On day 3 after surgery, a small number of X-galpositive cells emerged in the cambium layer of the periosteum adjacent to the fracture site (Fig. 5c-e). On day 5, X-gal-positive cells were located only in the soft callus and the thickened periosteum adjacent to the fracture site (Fig. 5f and g). These findings indicate that $\operatorname{Sox} 9$-expressing chondroprogenitor cells are induced in the periosteum adjacent to the fracture site.

Sox9-expressing cells in the periosteum predominantly contribute to the formation of the soft callus during fracture healing

To trace the fate of Sox9-expressing chondroprogenitor cells in the periosteum adjacent to the fracture site, we injected tamoxifen peritoneally into Sox9CreERT2;R26R mice for three days after surgery [11]. On day 3 after surgery and tamoxifen injection, no X-gal-positive cells were detected in the bones, including the periosteum, endosteum, and bone marrow (Fig. 6a and b). In contrast, the growth plate chondrocytes were X-gal positive. On day 5, X-gal-positive cells were detected in the thickened periosteum and the soft callus (Fig. 6c and d). On days 7 and 14, the cells in the soft and hard calli were X-gal positive (Fig. 6e-h). On day 28, X-gal-positive cells were detected in the newly formed periosteum and the remodeled bone tissues (Fig. 6i and j). These results indicate that the periosteal cells in which $\operatorname{Sox} 9$ expression was 
induced just after fracture gave rise to the chondrocytes and osteoblasts in the fracture callus and the cells in the newly formed periosteum during fracture healing.

\section{Discussion}

During the process of fracture healing, pluripotent mesenchymal progenitor cells must be recruited to the fracture site. These cells differentiate into chondrocytes and osteoblasts and contribute to the formation of the soft and hard calli. Several sources of these cells have been detected. Tavassoli and Crosby and Friedenstein et al. demonstrated the existence of mesenchymal stem cells in the bone marrow $[17,18]$. Farrington-Rock et al. and Brighton and Hunt reported that pericytes can potentially differentiate into chondrocytes [3, 19]. Lee et al. and Usas et al. showed evidence that muscle-derived cells adjacent to the bone tissues play a role in fracture healing because of their osteogenic potential $[5,20]$. Circulating progenitor cells in the bloodstream are also recruited to the fracture site through the stromal cell-derived factor-1/CXCR4 axis and develop a favorable environment for fracture healing by enhancing angiogenesis and osteogenesis [21]. However, when Colnot performed cell lineage analyses by transplanting bone grafts, she demonstrated that the bone itself is the main local source of cells for bone repair [7]. Osteoblasts and osteocytes originate from the periosteum, bone marrow, and endosteum, indicating that these three tissues contribute simultaneously to the formation of new bone. Chondrocytes within the fracture callus are primarily derived from the periosteum. This finding is supported by previous studies [22]. Periosteal damage or removal delays fracture healing because the local sources of pluripotent mesenchymal progenitor cells are thereby removed. 
The current mouse genetics approach based on the Cre/loxP recombination system allows us to define cell lineages at the cellular level. In this study, we first demonstrated that the osteochondroprogenitor cells in the periosteum make a distinct cellular contribution to the soft callus. Prxl is a homeobox-containing gene that starts to be expressed in all limb bud mesenchymal cells at E9.0, before $\operatorname{Sox} 9$ is expressed [12]. We have previously reported that conditional Sox9-null mutants harboring a Prx 1 -Cre transgene showed a complete lack of both cartilage and bone in their limbs [9]. Moreover, in Prxl-Cre;R26R embryos, all the cells in the skeletal elements, the fibroblasts in the connective tissues, and the tendon cells and the synovial cells were Xgal positive. Hence, Prxl-expressing undifferentiated limb bud mesenchymal cells, including Sox9-expressing osteochondroprogenitor cells, differentiate into all the mesenchymal cell populations. In $\operatorname{Prx} 1-C r e ; R 26 R$ mice, the periosteum, endosteum, and a small number of the cells in the bone marrow were X-gal positive, and almost all of the soft callus was also X-gal positive. This finding corresponds, in part, to that of Kawanami et al. in Prxl-CreER transgenic mice [23]. They found that Prxl-expressing periosteal cells differentiated into chondrocytes and osteoblasts in the fracture callus, further supporting the notion that $\operatorname{Prxl} 1$-expressing cells in the periosteum are osteochondroprogenitor cells. We also analyzed fracture healing in Collal(3.6 $\mathrm{kb}$ )Cre; 26 R mice. The $3.6-\mathrm{kb}$ Collal promoter is active in mesenchymal progenitor cells, immature preosteoblasts, and mature osteoblasts [24]. In the Collal(3.6 kb)-Cre;R26R mice, the cells in the periosteum, endosteum, and a small number of the cells in the bone marrow were $\mathrm{X}$-gal positive, and a proportion of the cells in the soft callus were $\mathrm{X}$-gal positive. In contrast, in Collal $(2.3 \mathrm{~kb})$-Cre; $R 26 R$ mice, a few cells in the periosteum and endosteum were X-gal positive because the $2.3-\mathrm{kb}$ Collal promoter is 
active in mature osteoblasts and osteocytes $[25,26]$, but no chondrocytes in the soft callus were $\mathrm{X}$-gal positive. The contribution to callus formation during fracture healing of cells that are Prxl-and Collal(3.6 kb)-promoter silent before fracture has not yet been clarified. However, our results indicate that a large proportion of chondrocytes in the soft callus at the fracture site are probably derived from cells originating from $\operatorname{Pr} x 1$ promoter-active mesenchymal progenitor cells in the bone tissue. Collal $(3.6 \mathrm{~kb})$ expressing cells in the periosteum also partly contribute to the formation of the soft callus.

We have previously shown that Sox9 defines osteochondroprogenitor cells [9]. In Sox9-LacZ mice, no cells in the periosteum express Sox9, suggesting that the pluripotent mesenchymal cells in the periosteum are not committed osteochondroprogenitor cells in the healthy condition. However, three days after a fracture occurs, the periosteal cells in the cambium layer adjacent to the fracture site transiently express Sox9. Therefore, we injected tamoxifen into Sox9-CreERT2;R26R mice for three days after fracture to label the $\operatorname{Sox} 9$-expressing periosteal cells and traced the fate of those cells. Interestingly, many X-gal-positive cells were detected in and around the soft and hard calli, indicating that the Sox9-expressing periosteal cells adjacent to the fracture site give rise to chondrocytes and osteoblasts in the fracture callus and in the newly formed periosteal tissues overlying the callus. Kawanami et al. showed that Prxl-expressing periosteal cells differentiate into undifferentiated cells, some of the chondrocytes in the soft callus, osteoblasts, and cells in the newly formed periosteum [23]. Taking all these findings together, pluripotent mesenchymal progenitor cells in the periosteum that originate from Prx 1-expressing mesenchymal cells give rise to Sox9-expressing osteochondroprogenitor cells during fracture healing, a process that 
recapitulates endochondral ossification in limb bud development. Sox9-expressing osteochondroprogenitor cells, possibly derived from Collal(3.6 $\mathrm{kb}$ )-expressing cells, emerged in the thickened periosteum adjacent to the fracture site and make a major contribution to the formation of the soft callus.

In conclusion, this cell lineage analysis using a mouse genetics approach has demonstrated that the periosteum plays a central role in soft callus formation during fracture healing. The pluripotent mesenchymal progenitor cells in the periosteum are committed to becoming Sox9-expressing osteochondroprogenitor cells shortly after a fracture occurs and differentiate into chondrocytes, osteoblasts, and periosteal cells. Further research is required to confirm how much Sox9-expressing osteochondroprogenitor cells contribute to fracture repair, and to isolate a pure population of Sox9-expressing cells from the periosteum, to determine the cellular and molecular characteristics of the periosteal osteochondroprogenitor cells.

\section{Conflict of interest}

All authors have no conflicts of interest.

\section{Acknowledgements}

We thank James F. Martin, Philippe Soriano, Chisa Shukunami, and Richard R. Behringer for their valuable help. This work was supported by Grants-in-Aid for Scientific Research from the Japanese Ministry of Education, Culture, Sports, Science, and Technology (\#21249078 and \#23659717 to HA and \#22791376 to KY). 
References

1. Squier CA, Ghoneim S, Kremenak CR (1990) Ultrastructure of the periosteum from membrane bone. J Anat 171:233-239

2. Schindeler A, McDonald MM, Bokko P, Little DG (2008) Bone remodeling during fracture repair: the cellular picture. Semin Cell Dev Biol 19:459-466

3. Farrington-Rock C, Crofts NJ, Doherty MJ, Ashton BA, Griffin-Jones C, Canfield AE (2004) Chondrogenic and adipogenic potential of microvascular pericytes. Circulation 110:2226-2232

4. Zuk PA, Zhu M, Mizuno H, Huang J, Futrell JW, Katz AJ, Benhaim P, Lorenz HP, Hedrick MH (2001) Multilineage cells from human adipose tissue: implications for cell-based therapies. Tissue Eng 7:211-228

5. Lee JY, Qu-Petersen Z, Cao B, Kimura S, Jankowski R, Cummins J, Usas A, Gates C, Robbins P, Wernig A, Huard J (2000) Clonal isolation of musclederived cells capable of enhancing muscle regeneration and bone healing. J Cell Biol 150:1085-1100

6. Kojimoto H, Yasui N, Goto T, Matsuda S, Shimomura Y (1988) Bone lengthening in rabbits by callus distraction. The role of periosteum and endosteum. J Bone Joint Surg Br 70:543-549

7. Colnot C (2009) Skeletal cell fate decisions within periosteum and bone marrow during bone regeneration. J Bone Miner Res 24:274-282 
8. Ito Y, Fitzsimmons JS, Sanyal A, Mello MA, Mukherjee N, O’Driscoll SW (2001) Localization of chondrocyte precursors in periosteum. Osteoarthritis Cartilage 9:215-223

9. Akiyama H, Chaboissier MC, Martin JF, Schedl A, de Crombrugghe B (2002) The transcription factor Sox9 has essential roles in successive steps of the chondrocyte differentiation pathway and is required for expression of Sox 5 and Sox6. Genes Dev 16:2813-2828

10. Akiyama H, Kim JE, Nakashima K, Balmes G, Iwai N, Deng JM, Zhang Z, Martin JF, Behringer RR, Nakamura T, de Crombrugghe B (2005) Osteochondroprogenitor cells are derived from Sox9 expressing precursors. Proc Natl Acad Sci U S A 102:14665-14670

11. Soeda T, Deng JM, de Crombrugghe B, Behringer RR, Nakamura T, Akiyama H (2010) Sox9-expressing precursors are the cellular origin of the cruciate ligament of the knee joint and the limb tendons. Genesis 48:635-644

12. Martin JF, Olson EN (2000) Identification of a prx1 limb enhancer. Genesis $26: 225-229$

13. Nakanishi R, Akiyama H, Kimura H, Otsuki B, Shimizu M, Tsuboyama T, Nakamura T (2008) Osteoblast-targeted expression of Sfrp4 in mice results in low bone mass. J Bone Miner Res 23:271-277 
14. Zhu J, Nakamura E, Nguyen MT, Bao X, Akiyama H, Mackem S. (2008) Uncoupling Sonic hedgehog control of pattern and expansion of the developing limb bud. Dev Cell 14:624-632

15. Soriano P (1999) Generalized lacZ expression with the ROSA26 Cre reporter strain. Nat Genet 21:70-71

16. Hogan B, Costantini F, Beddington R (1994) Manipulating the Mouse Embryo: A Laboratory Manual. Cold Spring Harbor Laboratory Press, New York

17. Tavassoli M, Crosby WH (1968) Transplantation of marrow to extramedullary sites. Science 161:54-56

18. Friedenstein AJ, Petrakova KV, Kurolesova AI, Frolova GP (1968) Heterotopic of bone marrow. Analysis of precursor cells for osteogenic and hematopoietic tissues. Transplantation 6:230-247

19. Brighton CT, Hunt RM (1997) Early histologic and ultrastructural changes in microvessels of periosteal callus. J Orthop Trauma 11:244-253

20. Usas A, Ho AM, Cooper GM, Olshanski A, Peng H, Huard J (2009) Bone regeneration mediated by BMP4-expressing muscle-derived stem cells is affected by delivery system. Tissue Eng Part A 15:285-293

21. Matsumoto T, Kawamoto A, Kuroda R, Ishikawa M, Mifune Y, Iwasaki H, Miwa M, Horii M, Hayashi S, Oyamada A, Nishimura H, Murasawa S, Doita M, Kurosaka M, Asahara T (2006) Therapeutic potential of vasculogenesis and 
osteogenesis promoted by peripheral blood CD34-positive cells for functional bone healing. Am J Pathol 169:1440-1457

22. Ozaki A, Tsunoda M, Kinoshita S, Saura R (2000) Role of fracture hematoma and periosteum during fracture healing in rats: interaction of fracture hematoma and the periosteum in the initial step of the healing process. J Orthop Sci 5:64-70

23. Kawanami A, Matsushita T, Chan YY, Murakami S (2009) Mice expressing GFP and CreER in osteochondroprogenitor cells in the periosteum. Biochem Biophys Res Commun 386:477-482

24. Boban I, Jacquin C, Prior K, Barisic-Dujmovic T, Maye P, Clark SH, Aguila HL (2006) The $3.6 \mathrm{~kb}$ DNA fragment from the rat Collal gene promoter drives the expression of genes in both osteoblast and osteoclast lineage cells. Bone $39: 1302-1312$

25. Dacic S, Kalajzic I, Visnjic D, Lichtler AC, Rowe DW (2001) Col1a1-driven transgenic markers of osteoblast lineage progression. J Bone Miner Res 16:12281236

26. Kalajzic I, Kalajzic Z, Kaliterna M, Gronowicz G, Clark SH, Lichtler AC, Rowe D (2002) Use of type I collagen green fluorescent protein transgenes to identify subpopulations of cells at different stages of the osteoblast lineage. J Bone Miner Res 17:15-25 
Figure legends

\section{Fig. 1}

A process of tibial fracture healing. Longitudinal sections through a mouse tibia stained with Alcian blue and hematoxylin/eosin. (a) Low magnification. (b) High magnification of the dotted box in (a). PO: periosteum; BM: bone marrow; SC: soft callus; HC: hard callus; RB: remodeled bone. Scale bar $=1 \mathrm{~mm}$ for (a) and $500 \mu \mathrm{m}$ for (b).

\section{Fig. 2}

Tibia fracture in $\operatorname{Prx} 1-C r e ; R 26 R$ mice. (a) X-gal-positive cells are found in the periosteum, cortical bone, and endosteum on day 0 . Scale bar $=1 \mathrm{~mm}$. (b) High magnification of (a). Arrowheads indicate X-gal-positive cells in the bone marrow. Scale bar $=100 \mu \mathrm{m}$. (c and e) Soft and hard calli are X-gal positive on days 7 and 14. (d and f) High magnification of (c) and (e). (g and i) Remodeled bone and the newly formed periosteum are X-gal positive on days 21 and 28. (h and j) High magnification of (g and i). MB: metaphyseal bone; GP: growth plate; PO: periosteum; EO: endosteum; BM: bone marrow; SC: soft callus; HC: hard callus; RB: remodeled bone. Scale bar = $1 \mathrm{~mm}$ for $(\mathrm{c}, \mathrm{e}, \mathrm{g}$, and i) and $500 \mu \mathrm{m}$ for $(\mathrm{d}, \mathrm{f}, \mathrm{h}$, and $\mathrm{j})$.

\section{Fig. 3}

Tibia fracture in Collal(3.6 kb)-Cre;R26R mice. (a) X-gal positive-cells are found in the periosteum, cortical bone, and endosteum on day 0 . Scale bar $=1 \mathrm{~mm}$. (b) High magnification of (a). Arrows indicate X-gal-positive cells in the periosteum and 
endosteum. Arrowheads indicate X-gal-positive cells in the bone marrow. Scale bar $=$ $100 \mu \mathrm{m}$. (c) Cells in the soft callus and the thickened periosteum adjacent to the fracture site are X-gal positive on day 7. (d and e) High magnification of the dotted bone and the solid box in (c). (f) Cells in the hard callus are X-gal positive on day 14. (g) High magnification of the dotted box in (f). (h and j) X-gal-positive cells are detected in the remodeled bone and the newly formed periosteum on days 21 and 28. (i) High magnification of the dotted box in (h). MB: metaphyseal bone; GP: growth plate; PO: periosteum; EO: endosteum; BM: bone marrow; SC: soft callus; HC: hard callus. Scale bar $=1 \mathrm{~mm}$ for $(\mathrm{c}, \mathrm{f}, \mathrm{h}$, and $\mathrm{j})$ and $500 \mu \mathrm{m}$ for $(\mathrm{d}, \mathrm{e}, \mathrm{g}$, and $\mathrm{i})$.

\section{Fig. 4}

Tibia fracture in Collal(2.3 kb)-Cre;R26R mice. (a) X-gal-positive cells are found in the periosteum, cortical bone, and endosteum on day 0. Scale bar $=1 \mathrm{~mm}$. (b) High magnification of (a). Scale bar $=100 \mu \mathrm{m}$. (c and e) Cells in the hard callus are X-gal positive on days 7 and 14. (d and f) High magnification of (c) and (e). (g and i) Cells in the remodeled bone are X-gal positive on days 21 and 28. (h and j) High magnification of (g) and (i). MB: metaphyseal bone; GP: growth plate; PO: periosteum; EO: endosteum; BM: bone marrow; SC: soft callus; HC: hard callus; RB: remodeled bone. Scale bar $=1 \mathrm{~mm}$ for $(c, e, g$, and i) and $500 \mu \mathrm{m}$ for $(\mathrm{d}, \mathrm{f}, \mathrm{h}$, and $\mathrm{j})$.

\section{Fig. 5}

Tibia fracture in Sox9-LacZ mice. (a) No X-gal-positive cells are found in the bones on day 0 . Scale bar $=1 \mathrm{~mm}$. (b) High magnification of (a). Growth plate chondrocytes are X-gal positive. Scale bar $=100 \mu \mathrm{m}$. (c) Low magnification of fractured tibia on day 3 . 
(d) High magnification of the dotted box in (c). No X-gal-positive cells are apparent around the fracture site. (e) High magnification of the dotted boxes in (d). A small number of X-gal-positive cells are found in the periosteum adjacent to the fracture site (arrowheads). (f) Low magnification of the fractured tibia on day 5. (g) High magnification of the dotted boxed in (f). All chondrocytic cells in the soft callus are Xgal positive. A large number of X-gal-positive cells are apparent in the thickened periosteum adjacent to the fracture site. MB: metaphyseal bone; GP: growth plate; PO: periosteum; EO: endosteum; BM: bone marrow; SC: soft callus. Scale bar $=1 \mathrm{~mm}$ for $(\mathrm{c}$, $\mathrm{d}$, and $\mathrm{f}$ ) and $100 \mu \mathrm{m}$ for (e and g).

\section{Fig. 6}

Tibia fracture in Sox9-CreERT2;R26R mice. The mice were injected with tamoxifen for three days after surgery. (a) No X-gal-positive cells are found in the bones on day 0 . Scale bar $=1 \mathrm{~mm}$. (b) High magnification of (a). Growth plate chondrocytes are X-gal positive. Scale bar $=100 \mu \mathrm{m}$. (c) Cells in the soft callus are X-gal positive on day 5. (d) High magnification of (c). (e and g) X-gal-positive cells are found in the soft and hard calli on days 7 and 14. (f and h) High magnification of the dotted boxes in (e) and (g). (i) The cells in the remodeled bone and the newly formed periosteum are positive for Xgal on day 28. (j) High magnification of the dotted box in (i). Arrowheads indicate Xgal-positive cells in the periosteum. MB: metaphyseal bone; GP: growth plate; PO: periosteum; EO: endosteum; BM: bone marrow; SC: soft callus; HC: hard callus; RB: remodeled bone. Scale bar $=1 \mathrm{~mm}$ for $(c, e, g$, and i) and $500 \mu \mathrm{m}$ for $(\mathrm{d}, \mathrm{f}, \mathrm{h}$, and $\mathrm{j})$. 


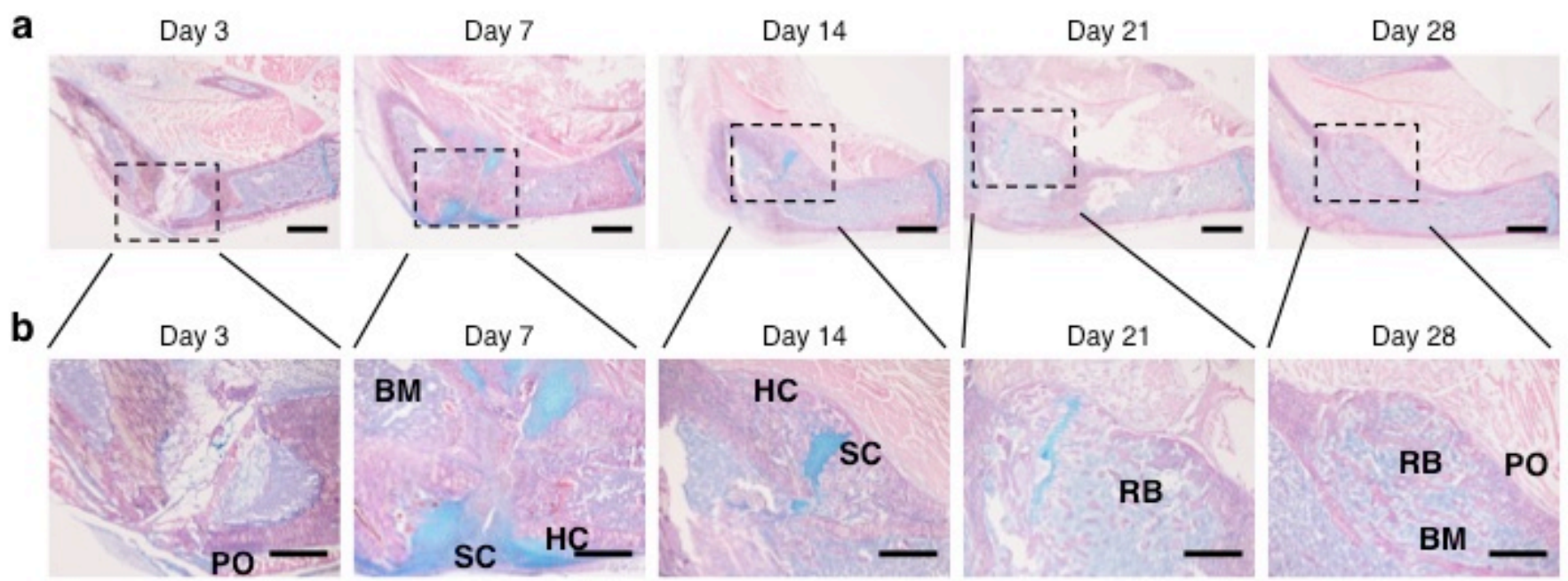

Fig. 1 
a

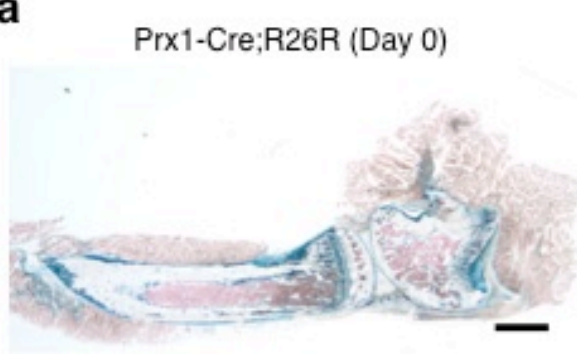

b
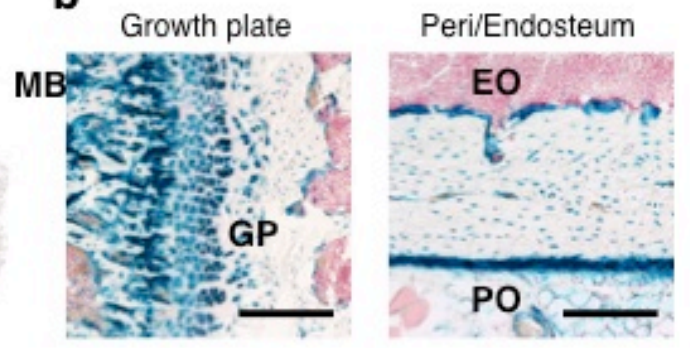

Bone marrow

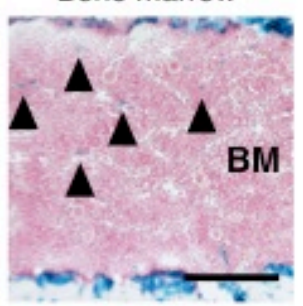

C

Day 7

e

Day 14

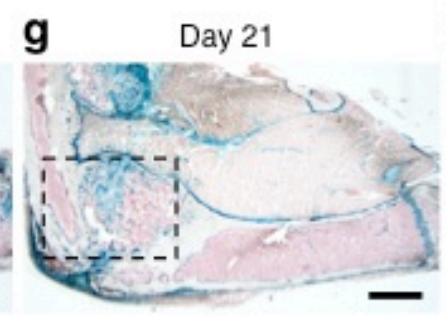

i

Day 28
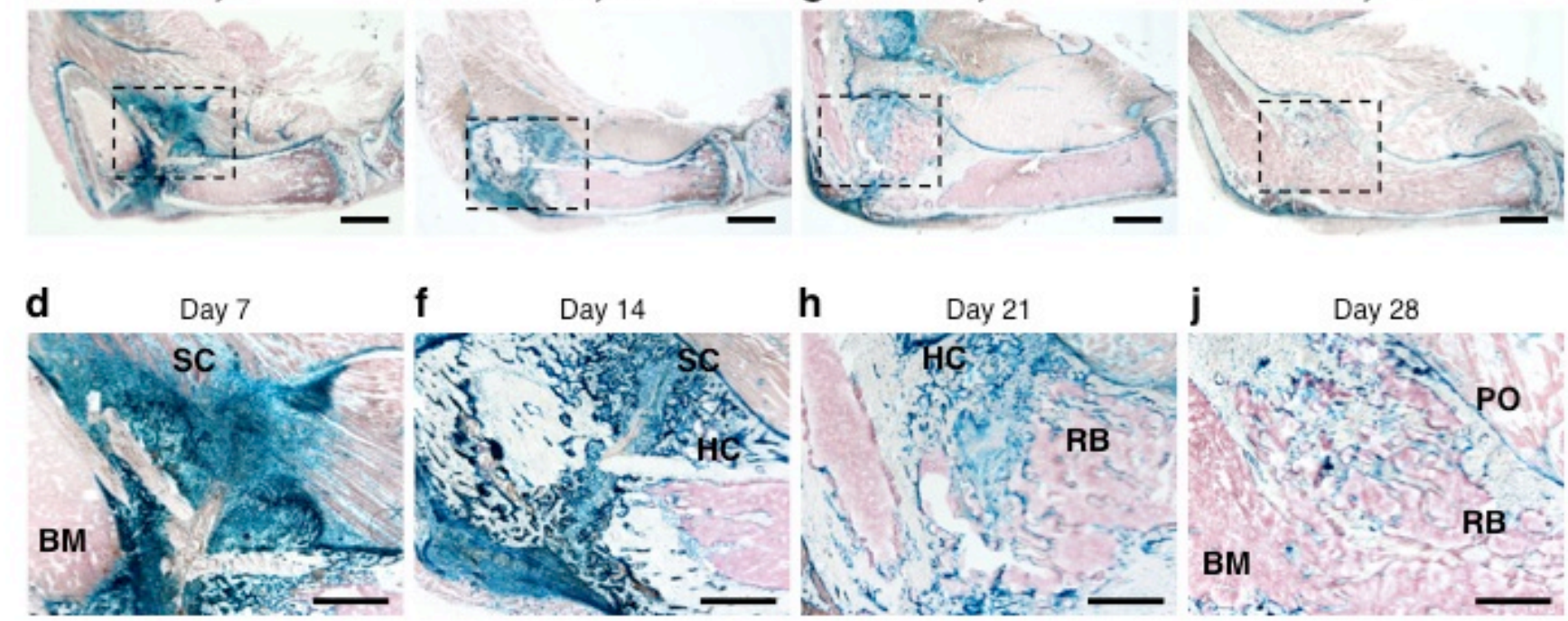

j Day 28

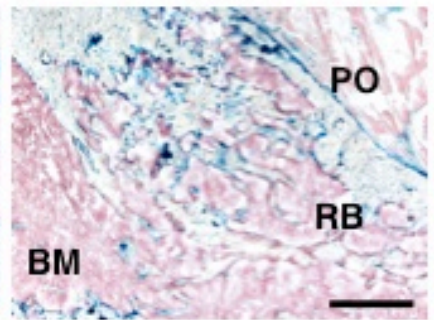

Fig. 2 
a Col1a1(3.6kb)-Cre;R26R (Day 0)

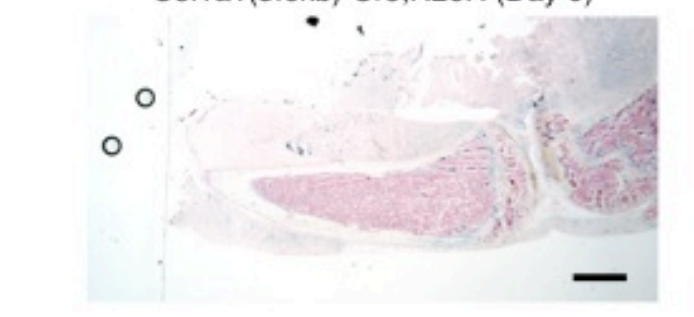

b

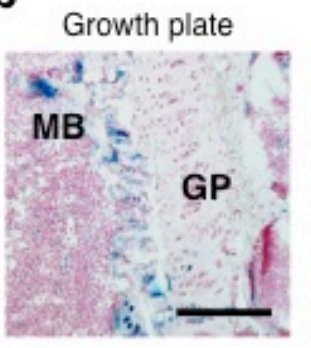

Peri/Endosteum

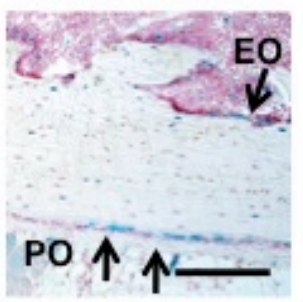

Bone marrow

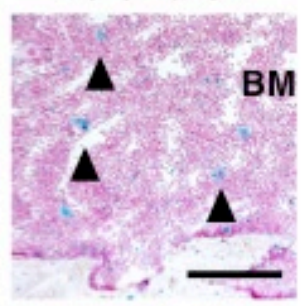

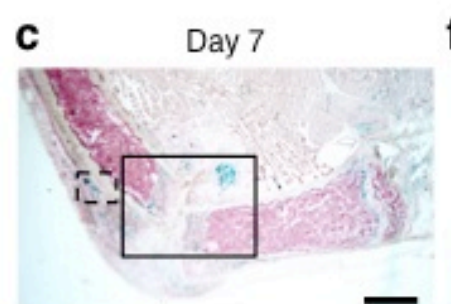

f Day 14

h

Day 21

j Day 28
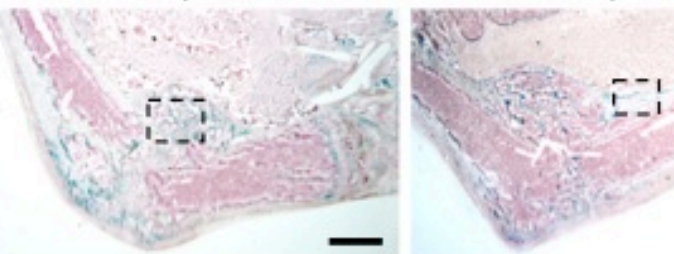

$--1$

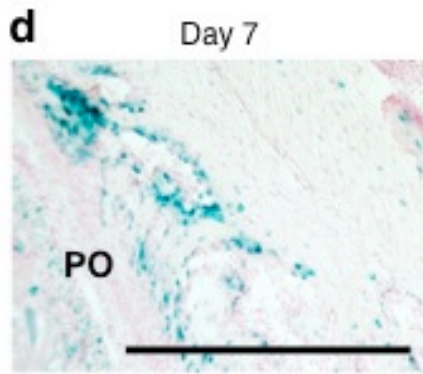

e $\quad$ Day 7

g

Day 14

i

Day 21

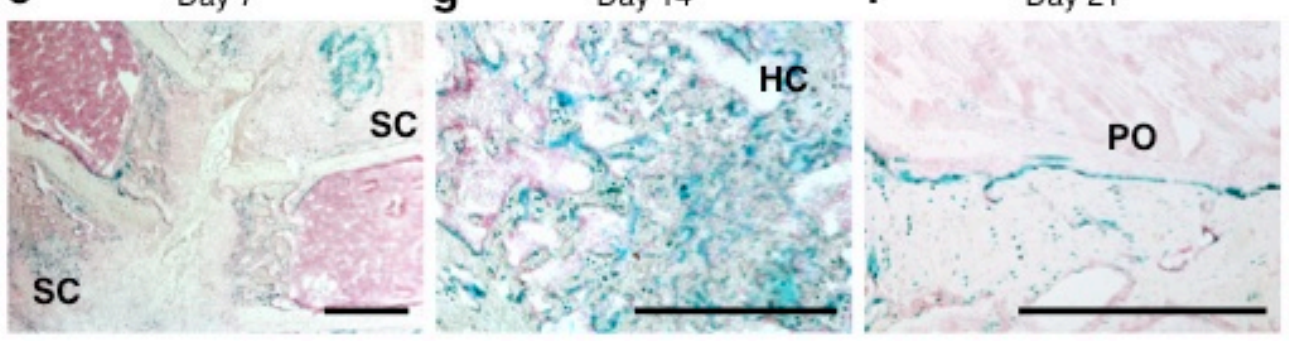

Fig. 3 
a

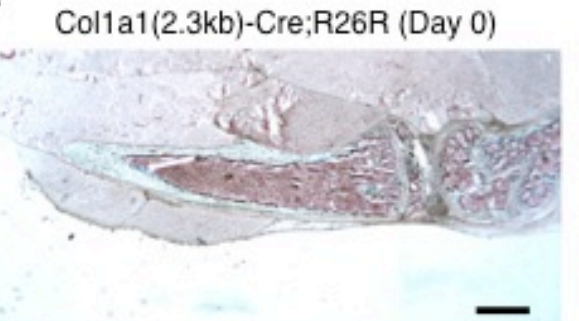

b

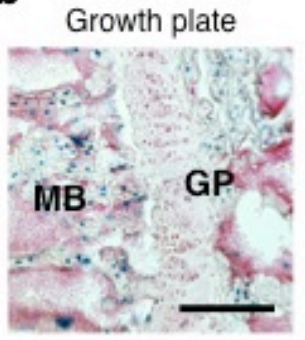

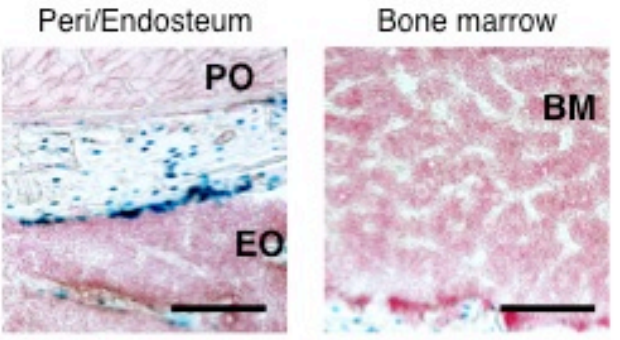

Day 21

i

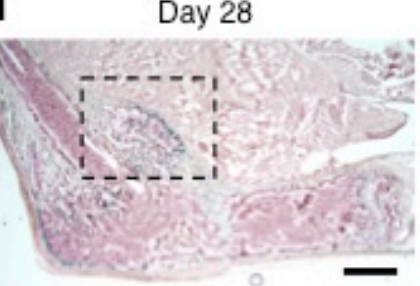

h

Day 21

j Day 28
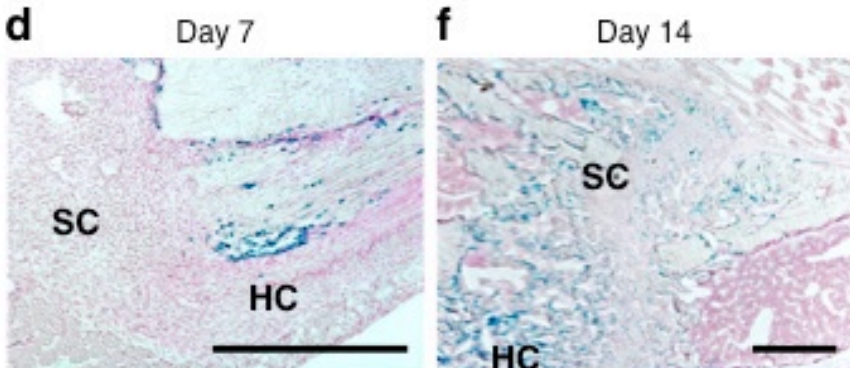

RB
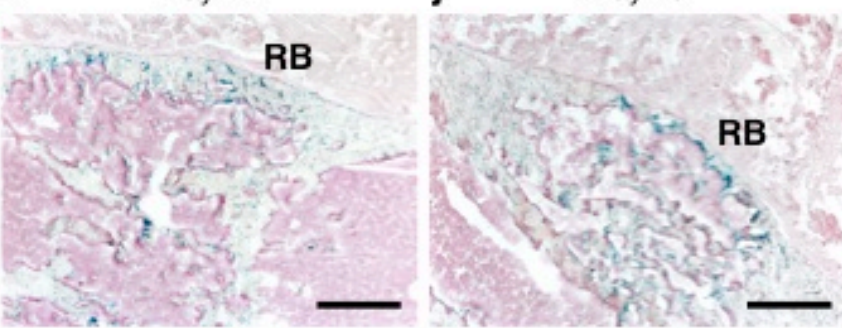

Fig. 4 
a

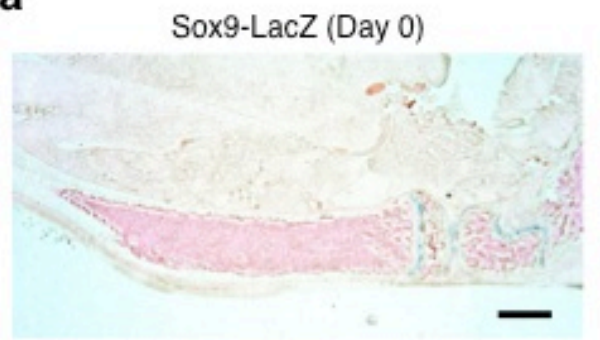

b

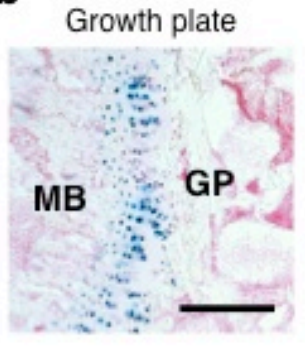

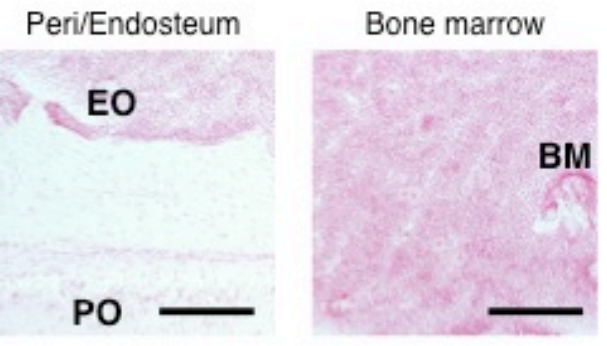

Periosteum
C

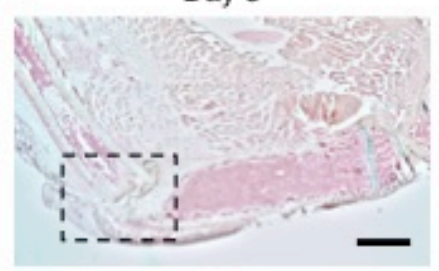

f

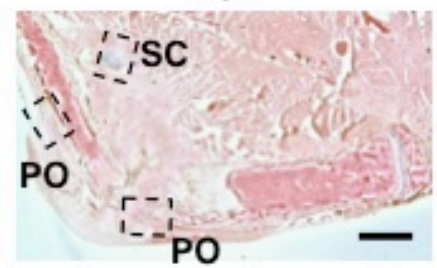

d Fracture site

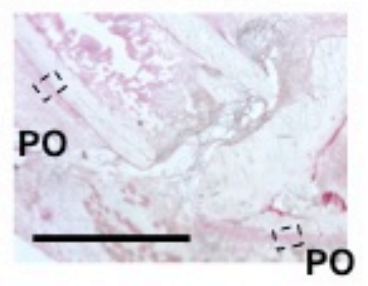

g

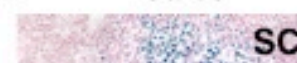

e

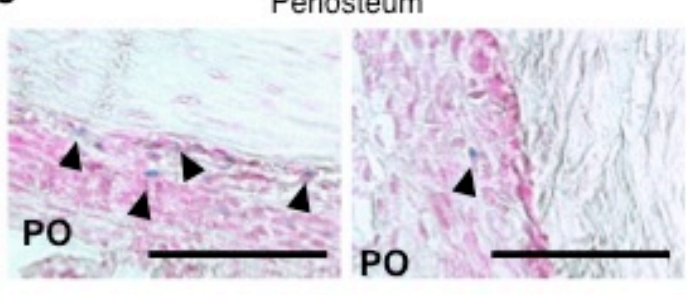

Periosteum

PO

- PO -

Fig. 5 
a

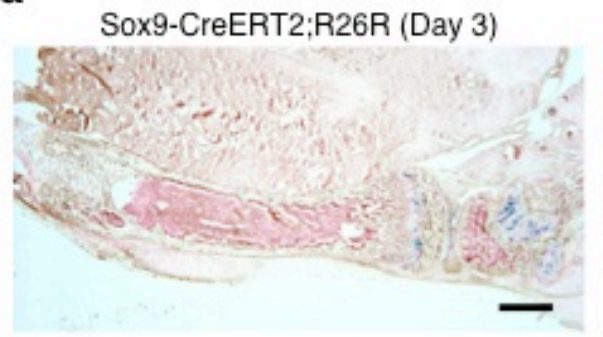

b

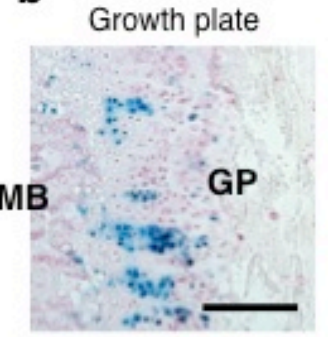

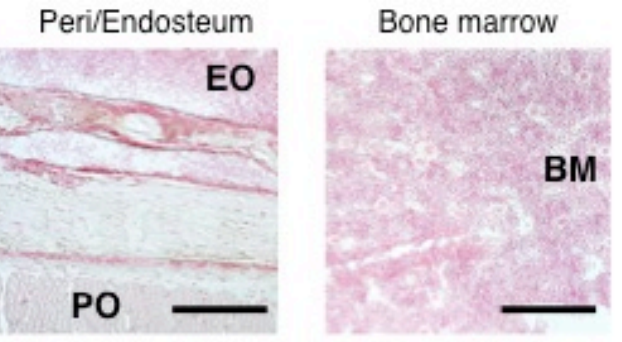

Day 28

Day 14

i

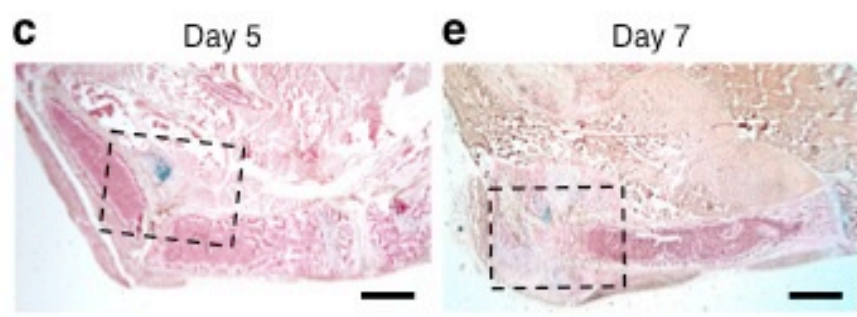

g
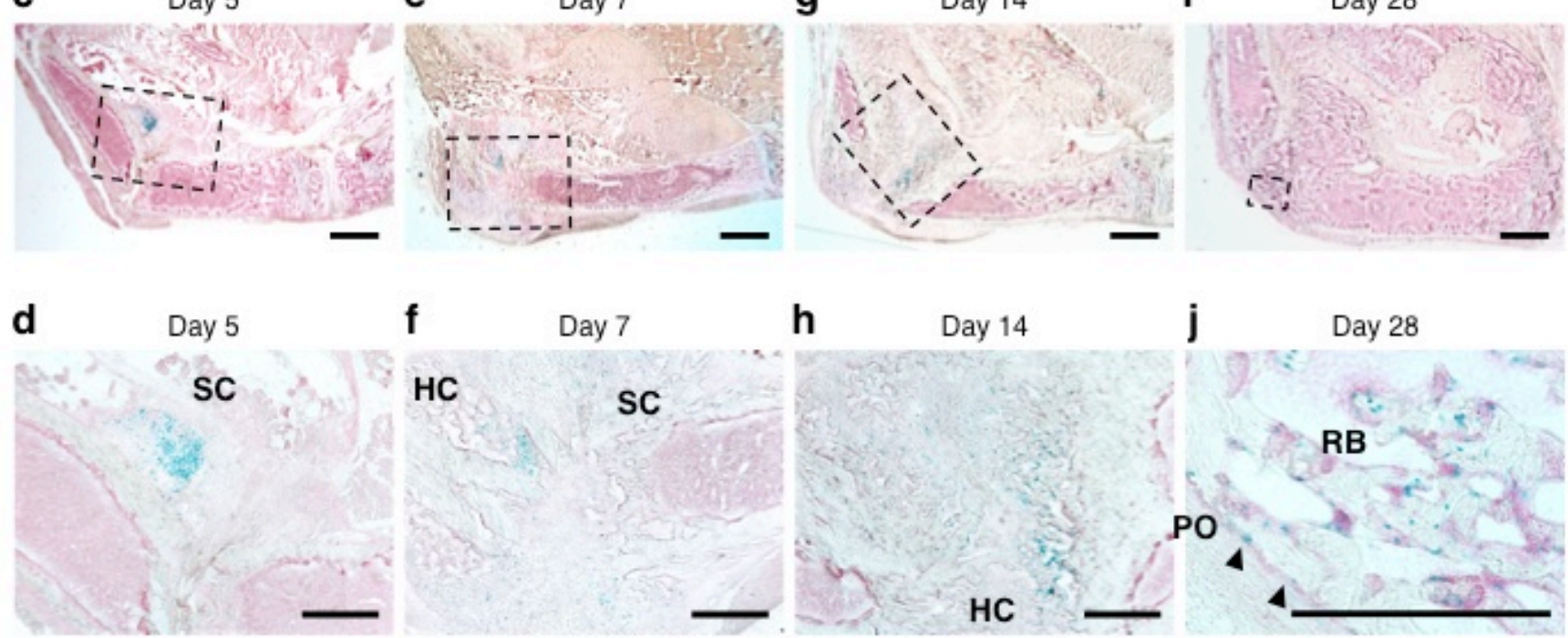

h

Day 14

j

Day 28

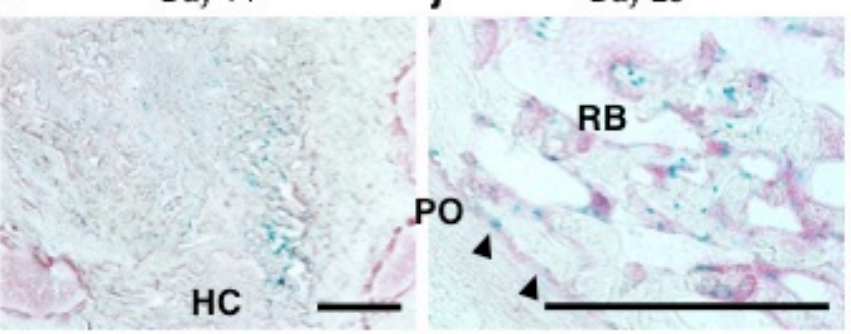

Fig. 6 